\title{
Micro-CT Analysis of Cranial Bone and Tooth Density in Mice Deficient for GDF11 or Myostatin
}

\author{
Joonho Suh, Na-Kyung Kim, Yun-Sil Lee \\ Department of Molecular Genetics \& Dental Pharmacology, School of Dentistry and Dental Research Institute, \\ Seoul National University, Seoul, Korea
}

Received October 18, 2020

Revised October 21, 2020

Accepted October 21, 2020
Correspondence to:

Yun-Sil Lee

Department of Molecular Genetics \& Dental Pharmacology, School of Dentistry and Dental Research Institute, Seoul National University, 101 Daehak-ro, Jongno-gu,

Seoul 03080, Korea

Tel: +82-2-880-2321

Fax: +82-2-882-0228

E-mail: yunlee@snu.ac.kr

https://orcid.org/0000-0002-1228-0404

This work was supported by CreativePioneering Researchers Program through Seoul National University (SNU), Basic Science Research Program through the National Research Foundation of Korea (NRF) funded by the Ministry of Education (NRF-2018R1D1A1B07045334), and the NRF grant funded by the Korea government (Ministry of Science and ICT) (NRF-

2020R1A2C1010359).
Purpose: Growth differentiation factor 11 (GDF11) and myostatin (MSTN) are closely-related transforming growth factor $\beta$ family members reported to play crucial roles in bone formation. We previously reported that, in contrast to MSTN, GDF11 promotes osteogenesis of vertebrae and limbs. GDF11 has been also reported as an important regulator in tooth development by inducing differentiation of pulp stem cells into odontoblasts for reparative dentin formation. The goal of this study was to investigate the differential roles of GDF11 and MSTN in dental and cranial bone formation.

Methods: Micro-computed tomography analysis was performed on cranial bones, including frontal, parietal, and interparietal bones, and lower incisors of wild-type, Gdf11 knockout $\left(G d f 11^{-1}\right)$, and Mstn knockout $\left(M_{s t n^{-1}}\right)$ mice. Tissue volume, thickness, and mineral density were evaluated for both cranial bone and lower incisors. Lower incisor lengths were also measured. Because $G d f 11^{-1-}$ mice die shortly after birth, analysis was performed on newborn (P0) mice.

Results: Compared to those of $M s n^{-1-}$ mice, cranial bone volume, thickness, and mineral density levels were all significantly diminished in $G d f 11^{-1-}$ mice. Tissue mineral density of $G d f 11^{-1-}$ mice were also significantly decreased compared to wild-type mice. Likewise, lower incisor length, tissue volume, thickness, and mineral density levels were all significantly reduced in $\mathrm{Gdfl1}^{-1_{-}^{-}}$mice compared to $\mathrm{Mstn}^{-1-}$ mice. Incisor length was also significantly decreased in $\mathrm{Gdfl1}^{-1-}$ mice compared to wild-type mice. $\mathrm{Mstn}^{-1-}$ mice exhibited mildly increased levels of tissue volume, thickness, and density in cranial bone and lower incisor compared to wild-type mice although statistically not significant.

Conclusions: Our findings suggest that GDF11, unlike MSTN, endogenously promotes cranial bone and tooth development.

Key Words: Cranial bone; GDF11; Micro-computed tomography; Myostatin; Tooth

\section{INTRODUCTION}

Members of the transforming growth factor $\beta$ (TGF $\beta$ ) family, such as activins, growth differentiation factors (GDFs), bone morphogenetic proteins (BMPs), and TGF $\beta$ s, have been widely implicated to play critical roles during skeletal, craniofacial, and dental tissue development [1].
GDF11, also known as BMP11, and myostatin (MSTN) are closely-related TGF $\beta$ family members that share $89 \%$ amino acid sequence identity in their mature domain [2]. Despite sharing high sequence similarity, GDF11 and MSTN exert distinct endogenous functions. Mstn is first expressed in developing somites and continues to be expressed in skeletal muscle tissues to suppress skeletal muscle development

Copyright (c) 2020 Korean Academy of Orofacial Pain and Oral Medicine. All rights reserved.

(c) This is an open-access article distributed under the terms of the Creative Commons Attribution Non-Commercial License (http://creativecommons.org/licenses/by-nc/4.0/), which permits unrestricted non-commercial use, distribution, and reproduction in any medium, provided the original work is properly cited. 
[3]. Mice homozygous for Mstn deletion exhibit doubling of skeletal muscle mass throughout the body and elevated bone mineral density [3-6]. Mstn-null mice also display significantly increased temporalis, masseter, pterygoid muscles, and bite forces [7], and morphological analysis of the skulls in these mice revealed that they have shorter cranial vault and longer cranial base than wild-type mice [8-10], although no difference in cranial length, width, height, and volume between Mstn-null and wild-type mice has also been reported [11]. More recent analysis demonstrated that endocranial volume is reduced in adult Mstn-deficient mice potentially due to the influence of enlarged masticatory muscles [12]. Furthermore, adult Mstn-deficient mice were shown to exhibit significantly enhanced levels of incisor dentin mineral density [13].

Gdf11 is expressed in the tail bud during embryonic development and controls axial skeletal and organ patterning [2]. Gdf11-null mice display anterior homeotic transformation of the vertebrae, lack of tail, and defects in organ development $[2,14,15]$. GDF11 also regulates proper craniofacial development, as Gdf11-deficient mice experience cleft palate with high (60\%) penetrance [16]. Recently, a family with orofacial clefting and vertebral/rib hypersegmentation was identified to carry a missense mutation in Gdf11 [17], indicating that the crucial developmental function of GDF11 translates to humans. GDF11, which is highly expressed in differentiated odontoblasts [18], was also demonstrated to stimulate tooth development by inducing differentiation of pulp stem cells into odontoblasts, and electroporationmediated delivery of Gdf11 gene promoted reparative dentin formation in the amputated pulp of canine teeth $[19,20]$. Furthermore, mice lacking both Gdf11 and Gasp1, an endogenous inhibitor of GDF11 and MSTN, exhibited underdeveloped or missing tooth buds for lower incisors [21].

Because GDF11 and MSTN have been suggested to play significant roles during craniofacial and dental tissue development, targeting these molecules may have the potential to become an effective therapeutic strategy for tissue regeneration; however, their exact functions in craniofacial and tooth development require further investigation. Recently, we reported that GDF11 and MSTN play opposite roles during vertebral and limb bone development [4]. The goal of this study was to investigate the roles of GDF11 and MSTN in cranial bone and tooth formation, and whether GDF11 and MSTN also play differential functions in these tissues. Because micro-computed tomography (micro-CT) imaging is a relatively inexpensive and simple method that provides high throughput analysis with high resolution, making it ideal for newborn mice phenotyping especially in hard tissues such as bone and tooth, here, we used micro-CT analysis to evaluate cranial bones and lower incisors of mice deficient for Gdf11 or Mstn in order to examine whether GDF11 and MSTN also exert divergent functions in these tissues.

\section{MATERIALS AND METHODS}

\section{Mice}

All animal studies were approved by the Institutional Animal Care and Use Committees at Seoul National University. Gdf11 homozygous knockout $\left(G d f 11^{-1}\right)$ and Mstn homozygous knockout $\left(\mathrm{Mstn}^{-1-}\right)$ mice were kindly provided by Dr. Se-Jin Lee at Johns Hopkins University (currently at University of Connecticut School of Medicine). Msth $^{-1-}$ mice were viable and fertile, but Gdf11 ${ }^{-/}$mice died within one day after birth. Due to the perinatal lethality of $G d f 11^{-/-}$ mice, the head samples for the cranial bone and tooth density measurement were collected from newborn (P0) mice. All mice were maintained on a C57BL/6 background.

\section{Micro-computed tomography}

Micro-CT analysis was performed using Skyscan 1,172 and 1,272 (Bruker-MicroCT) following the manufacturers' guidelines (provided by Bruker). Skyscan 1,272 was used to generate representative images of calvarial bones, which were taken with a pixel size of $8 \mu \mathrm{m}$ at $60 \mathrm{kV}$ and $166 \mu \mathrm{A}$ through $0.25 \mathrm{~mm}$ aluminum filter. Skyscan 1,172 was used to generate representative images of lower incisors, which were taken with a pixel size of $4 \mu \mathrm{m}$ at $50 \mathrm{kV}$ and $200 \mu \mathrm{A}$ through no filter. Morphometric analyses of cranial bone and lower incisors were performed on samples scanned with Skyscan 1,272 with a pixel size of $8 \mu \mathrm{m}$ at $60 \mathrm{kV}$ and $166 \mu \mathrm{A}$ through $0.25 \mathrm{~mm}$ aluminum filter. Region of interests for cranial bones and lower incisors were extracted and analyzed using CTAn (v1.17.7.2; Bruker-MicroCT, Konitich, Belgium), a manufacturer-provided software. Lower incisor 
length was measured using the Dataviewer (v1.5.6.2; Bruker-MicroCT) software. Images were reconstructed and displayed using manufacturer-provided software NRecon (v1.7.3.2; Bruker-MicroCT), CTvox (v3.3.0; Bruker-MicroCT), and CTvol (v2.3.2.0; Bruker-MicroCT).

\section{Statistical analysis}

All values are presented as mean \pm standard error of the mean (SEM) from at least three independent experiments unless otherwise stated. One-way ANOVA with Tukey's post hoc test was performed for statistical analyses. A $\mathrm{p}<0.05$ was considered significant.

\section{RESULTS}

Because Gdf11-null mice are perinatally lethal in contrast to Mstn-null mice that survive into adulthood, we analyzed the tissues of newborn (P0) mice. We first examined the flat bones of the cranium, including the frontal, parietal, and interparietal bones of newborn wild-type, $G d f 11^{-1}$, and $\mathrm{Mstn}^{-1-}$ mice using micro-CT (Fig. 1). Analysis indicated that $G d f 11^{-1-}$ mice clearly displayed retarded ossification as described in the three-dimensional reconstructed images of calvarial bones (Fig. 1A). Color-coded images showing the distribution of cranial bone thickness also demonstrated delayed ossification in both frontal and parietal bones of Gdf11 ${ }^{-1-}$ mice (Fig. 1A). In contrast to $\mathrm{Gdf11^{-1 }}$ mice, $\mathrm{Mstn}^{-1-}$ mice displayed the greatest calvarial ossification progression among all groups and enhanced levels of bone thickness particularly in the frontal bone area as shown in the color-coded image (Fig. 1A). Assessment of bone morphology revealed that compared to those of $\mathrm{Mstn}^{-1-}$ mice, cranial bone volume, thickness, and tissue mineral density levels were all significantly diminished in $G d f 11^{-1-}$ mice (Fig. 1B). Tissue mineral density of $G d f 11^{-1-}$ mice were also significantly decreased compared to wild-type mice (Fig. 1B).
A

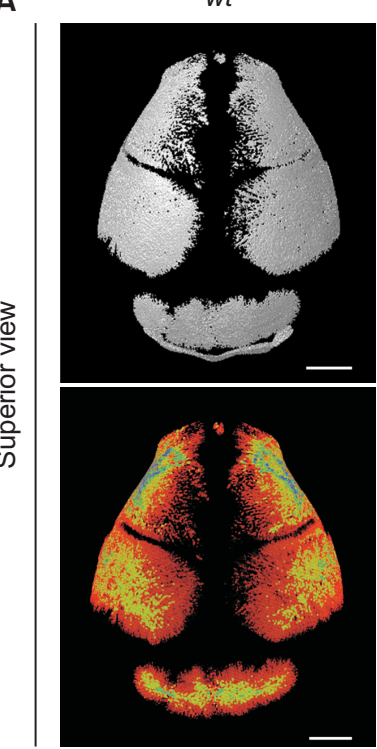

B

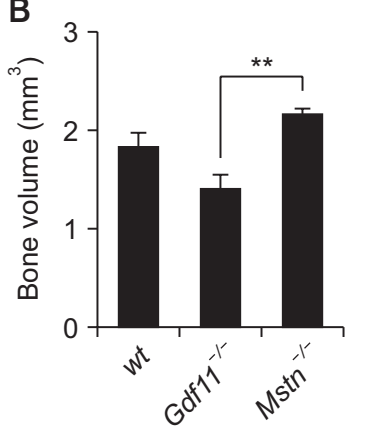

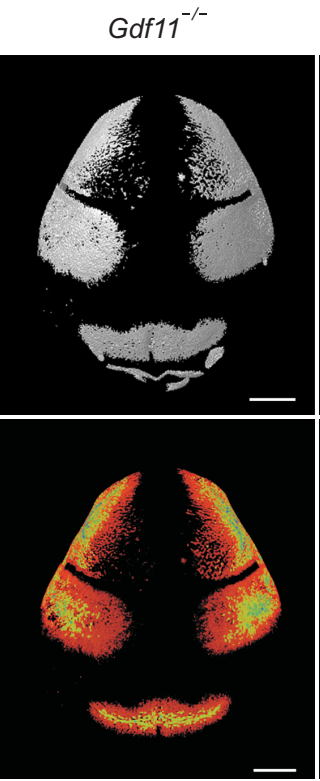

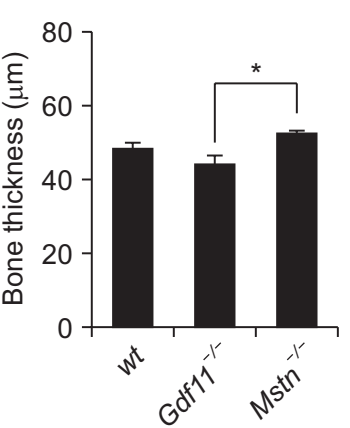

$\mathrm{Mstn}^{-/-}$
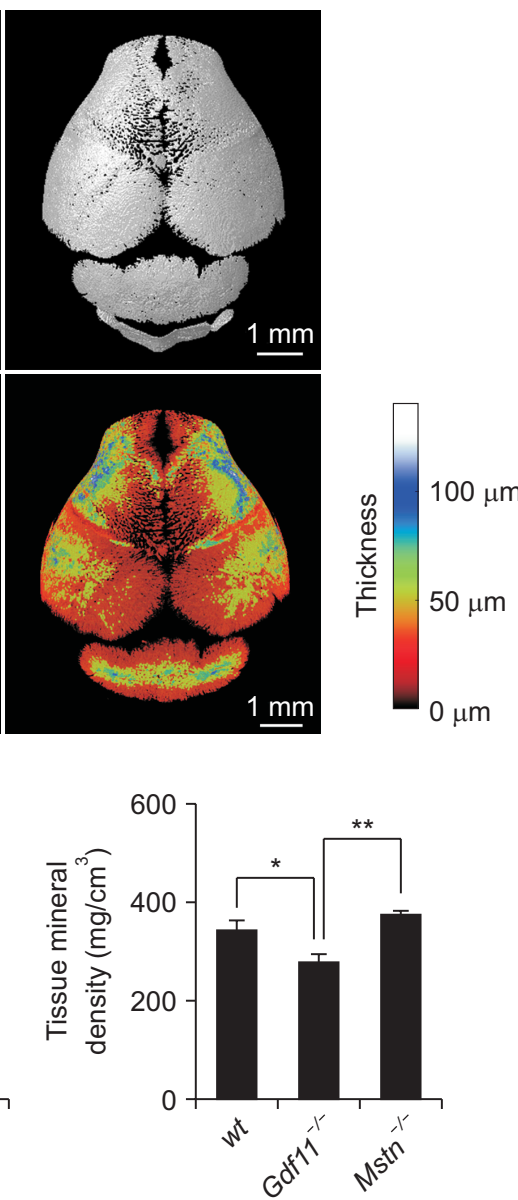

Fig. 1. GDF11 deficiency, in contrast to MSTN deficiency, impairs calvarial bone formation. (A) Representative threedimensional reconstructed micro-CT images of calvarial bones of newborn wt, Gdf11 ${ }^{-1-}$, and Mstn ${ }^{-1-}$ mice. Superior views are displayed, and color-coded images that represent bone thickness distribution are shown in the lower panel. Note the retarded ossification in $\mathrm{Gdfl1}^{-1-}$ mice. All scale bars are displayed with actual size values. (B) Morphometric analysis of cranial bone volume, bone thickness, and tissue mineral

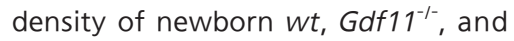
$\mathrm{Mstn}^{-1-}$ mice ( $\mathrm{n}=4$ mice each). GDF11, growth differentiation factor 11; MSTN, myostatin; Micro-CT, micro-computed tomography; wt, wild-type. All data represent mean \pm standard error of the mean. ${ }^{*} p<0.05,{ }^{* *} p<0.01$ by ANOVA with Tukey's post hoc test. 
Although statistically not significant, Mstn $^{-1-}$ mice exhibited elevated levels of bone volume, thickness, and density compared to wild-type mice (Fig. 1B). These results suggest that endogenous GDF11 promotes cranial bone development in contrast to MSTN.

Gdf11 gene delivery was reported to stimulate tooth development by inducing odontoblast differentiation of dental pulp cells [20]. However, the reports on the analysis of dental tissues in $\mathrm{Gdf11}^{-{ }_{-}}$mice are lacking, although we previously demonstrated an analysis performed in Gasp ${ }^{1--}$; $\mathrm{Gdf11}^{-1_{-}^{-}}$mice [21]. Using micro-CT, we evaluated the lower

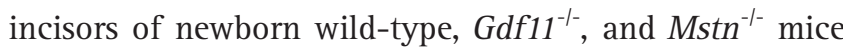
(Fig. 2A). Comparisons of midsagittal and parasagittal views of lower incisors between these mice showed that incisor development was substantially impaired in $G d f 11^{-1-}$ mice that displayed the smallest incisor size (Fig. 2B). Mandibular bone of $G d f 11^{-1-}$ mice also appeared to be the least dense among all groups, although quantifications were not performed (Fig. 2B). Likewise, color-coded images that represent tissue thickness showed that thickness levels were noticeably reduced in $G d f 11^{-1-}$ mice particularly in the midsagittal region of the incisor (Fig. 2B). Morphometric analysis revealed that lower incisor length, tissue volume, thickness, and mineral density levels were all significantly diminished in $\mathrm{Gdfl1}^{-1_{-}^{-}}$mice compared to $\mathrm{Mstn}^{-/-}$mice (Fig. 2C). Lower incisor length were also significantly decreased
A

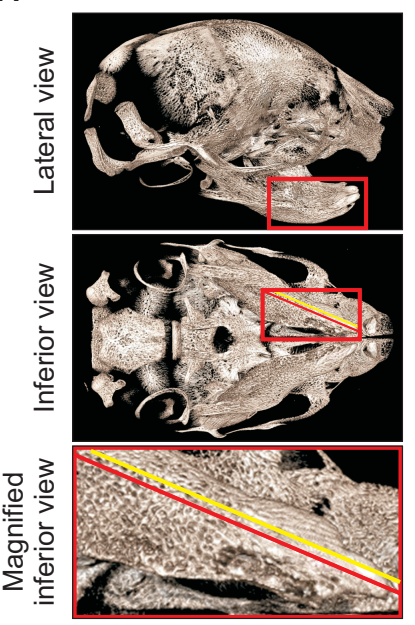

B

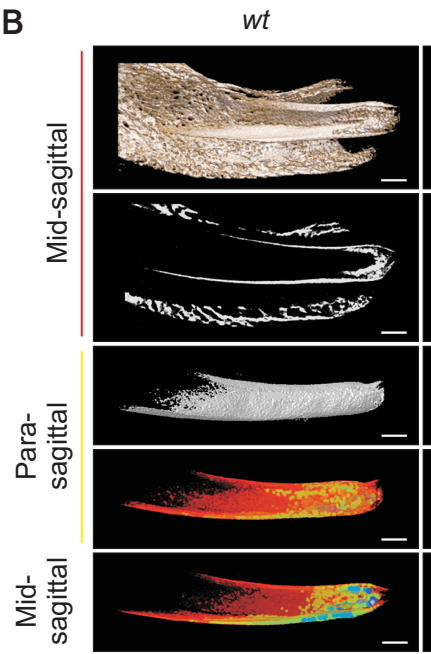

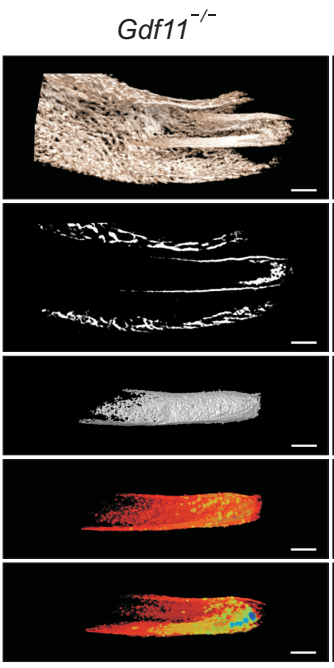
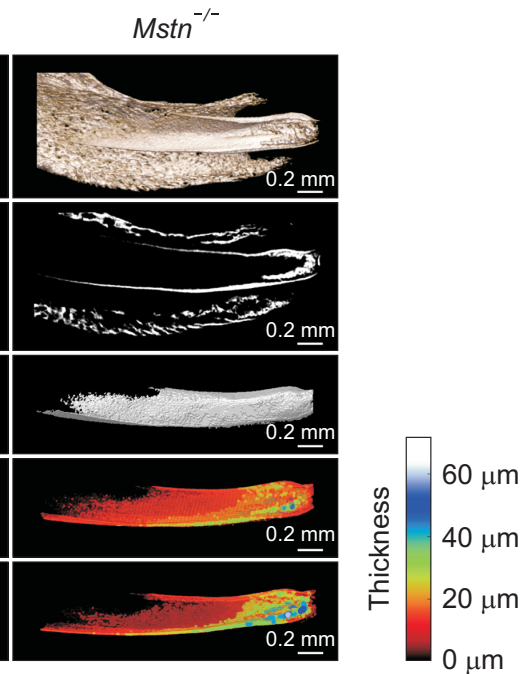

C
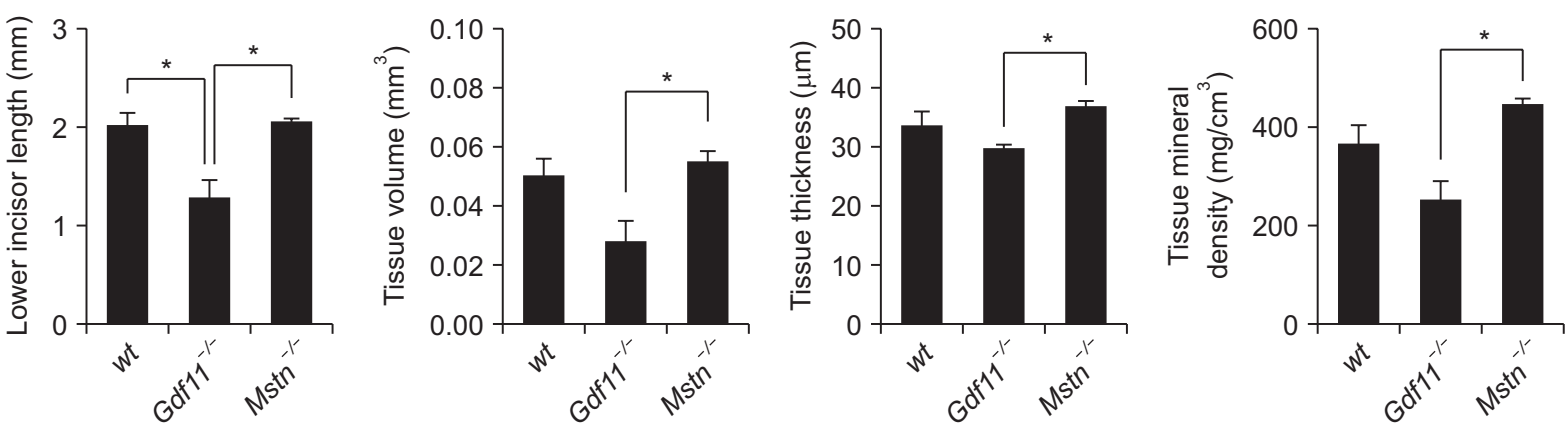

Fig. 2. GDF11 deficiency impairs lower incisor formation. (A) Reconstructed micro-CT images of newborn mouse skull. The top and middle panels represent lateral and inferior views, respectively. The red boxed region in the top panel indicates the area displayed in (B). Magnified inferior view of the red boxed region in the middle panel is displayed in the bottom panel. The red and yellow lines indicate the midsagittal and parasagittal planes of the lower incisor, respectively. (B) Representative three-dimensional reconstructed micro-CT images of lower incisors of newborn wt, Gdf11 ${ }^{-1-}$, and $\mathrm{Mstn}^{-1-}$ mice. Color-coded images that represent tissue thickness distribution are shown in the lower panels. Note the reduced incisor length in $\mathrm{Gdf11^{-1- }}$ mice. All scale bars are displayed with actual size values. (C) Morphometric analysis of lower incisor length, tissue volume, tissue thickness, and tissue mineral density of newborn wt, Gdf11 ${ }^{-1-}$, and Mstn ${ }^{-1-}$ mice ( $\mathrm{n}=3$ mice each). GDF11, growth differentiation factor 11; Micro-CT, micro-computed tomography; wt, wild-type; MSTN, myostatin. All data represent mean \pm standard error of the mean. ${ }^{*} \mathrm{p}<0.05$ by ANOVA with Tukey's post hoc test. 
in $\mathrm{Gdf}_{11^{-1}}$ mice compared to wild-type mice (Fig. 2C). No difference in incisor length was observed between wild-type and $\mathrm{Mstr}^{-1-}$ mice, although tissue thickness and mineral density appeared to be mildly greater in $\mathrm{Mstn}^{-1-}$ mice (Fig. 2C). These results indicate that GDF11 endogenously promotes tooth development as opposed to MSTN.

\section{DISCUSSION}

GDF11 and MSTN, which share high amino acid sequence identity, play critical, but distinct roles during embryonic development. While MSTN is a potent inhibitor of skeletal muscle growth [3], GDF11 regulates axial skeletal patterning, organ development, and palatal formation [16]. We previously showed that GDF11, as opposed to MSTN, promotes osteogenesis of the vertebrae and limbs that are developed through endochondral ossification [4]. Here, we focused on the evaluation of membranous ossification of cranial bones in wild-type, Gdf11 ${ }^{-1-}$, and $\mathrm{Mstn}^{-{ }^{--}}$mice and demonstrated that GDF11 and MSTN also exert opposite functions in cranial bone development. Lack of GDF11 resulted in a clear suppression of ossification, implying that GDF11 endogenously promotes calvarial bone formation. We further analyzed lower incisors of wild-type, Gdf1 $11^{-1-}$, and $\mathrm{Mstn}^{-1-}$ mice and showed that GDF11 also endogenously stimulates tooth development unlike MSTN.

Although GDF11 and MSTN play divergent roles, the existence of some functional redundancy in regulating axial skeletal patterning and cranial development has been reported, as demonstrated by more severe homeotic transformations of Gdf11 and Mstn double-mutants compared to Gdf11 ${ }^{-1}$ mice, and rounded frontal bone observed only in the double-mutant mice [22]. However, our previous analysis revealed that loss of Gasp1, an endogenous inhibitor of GDF11 and MSTN, in Gdf11 ${ }^{-1-}$ mice, which is expected to increase MSTN activity, considerably elevated the occurrence of dental malformation and cleft palate [21], adding further complexity to the regulation of craniofacial development by GDF11 and MSTN.

Whether the divergent functions of GDF11 and MSTN simply reflect their distinct expression patterns or reflect differences in their signaling mechanisms remains to be clarified. Both the mature GDF11 and MSTN initially bind to activin type 2 receptors (ACVR2A or ACVR2B) and recruit type 1 receptors (ALK4 or ALK5) to generate downstream signal transduction through SMAD2/3 phosphorylation [23]. More recently, studies have reported that GDF11 can potently activate BMP signaling, phosphorylating SMAD1/5/9 in endothelial cells and calvarial osteoblasts [4,24-26]. Because BMP signaling in differentiated odontoblasts is critical for dentin production in teeth [27], and Gdf11 gene therapy in pulp was shown to induce dentin matrix synthesis as observed with BMP2 and BMP4 protein therapy [28], endogenous GDF11 may activate BMP signaling pathway in odontoblasts to promote tooth development, although direct examination is necessary to confirm this possibility.

Understanding the pathogenesis of craniofacial abnormalities and dysmorphic tooth formation is essential for the improvement of dental treatment options for these defects. Our results demonstrate that GDF11, unlike MSTN, is an endogenous promoter of cranial bone formation and tooth development. However, due to the perinatal lethality of $G d f 11^{-1}$ mice, our analysis was limited to newborn mice. Future examination should utilize conditional knockout techniques in mice to elucidate the postnatal functions of GDF11 in craniofacial and tooth development.

\section{CONFLICT OF INTEREST}

No potential conflict of interest relevant to this article was reported.

\section{ORCID}

\author{
Joonho Suh \\ https://orcid.org/0000-0003-2438-0071 \\ Na-Kyung Kim \\ https://orcid.org/0000-0001-7675-5141 \\ Yun-Sil Lee \\ https://orcid.org/0000-0002-1228-0404
}

\section{REFERENCES}

1. Dudas M, Kaartinen V. Tgf-beta superfamily and mouse craniofacial development: interplay of morphogenetic proteins and receptor signaling controls normal formation of the face. Curr Top Dev Biol 2005;66:65-133. 
2. McPherron AC, Lawler AM, Lee SJ. Regulation of anterior/posterior patterning of the axial skeleton by growth/differentiation factor 11. Nat Genet 1999;22:260-264.

3. McPherron AC, Lawler AM, Lee SJ. Regulation of skeletal muscle mass in mice by a new TGF-beta superfamily member. Nature 1997;387:83-90.

4. Suh J, Kim NK, Lee SH, et al. GDF11 promotes osteogenesis as opposed to MSTN, and follistatin, a MSTN/GDF11 inhibitor, increases muscle mass but weakens bone. Proc Natl Acad Sci U S A 2020;117:4910-4920.

5. Nicholson EK, Stock SR, Hamrick MW, Ravosa MJ. Biomineralization and adaptive plasticity of the temporomandibular joint in myostatin knockout mice. Arch Oral Biol 2006;51:37-49.

6. Hamrick MW. Increased bone mineral density in the femora of GDF8 knockout mice. Anat Rec A Discov Mol Cell Evol Biol 2003;272:388-391.

7. Williams SH, Lozier NR, Montuelle SJ, de Lacalle S. Effect of postnatal myostatin inhibition on bite mechanics in mice. PLoS One 2015;10:e0134854.

8. Vecchione L, Miller J, Byron C, et al. Age-related changes in craniofacial morphology in GDF-8 (myostatin)-deficient mice. Anat Rec (Hoboken) 2010;293:32-41.

9. Byron CD, Maness H, Yu JC, Hamrick MW. Enlargement of the temporalis muscle and alterations in the lateral cranial vault. Integr Comp Biol 2008;48:338-344.

10. Vecchione L, Byron C, Cooper GM, et al. Craniofacial morphology in myostatin-deficient mice. J Dent Res 2007;86:1068-1072.

11. Cray J Jr, Kneib J, Vecchione L, et al. Masticatory hypermuscularity is not related to reduced cranial volume in myostatinknockout mice. Anat Rec (Hoboken) 2011;294:1170-1177.

12. Jeffery N, Mendias C. Endocranial and masticatory muscle volumes in myostatin-deficient mice. R Soc Open Sci 2014;1:140187.

13. Ravosa MJ, Klopp EB, Pinchoff J, Stock SR, Hamrick MW. Plasticity of mandibular biomineralization in myostatin-deficient mice. J Morphol 2007;268:275-282.

14. Harmon EB, Apelqvist AA, Smart NG, Gu X, Osborne DH, Kim SK. GDF11 modulates NGN3+ islet progenitor cell number and promotes beta-cell differentiation in pancreas development. Development 2004;131:6163-6174.

15. Esquela AF, Lee SJ. Regulation of metanephric kidney development by growth/differentiation factor 11. Dev Biol 2003;257:356370.

16. Suh J, Eom JH, Kim NK, et al. Growth differentiation factor 11 lo- cally controls anterior-posterior patterning of the axial skeleton. J Cell Physiol 2019;234:23360-23368.

17. Cox TC, Lidral AC, McCoy JC, et al. Mutations in GDF11 and the extracellular antagonist, Follistatin, as a likely cause of Mendelian forms of orofacial clefting in humans. Hum Mutat 2019;40:1813-1825.

18. Nakashima M, Toyono T, Akamine A, Joyner A. Expression of growth/differentiation factor 11, a new member of the BMP/ TGFbeta superfamily during mouse embryogenesis. Mech Dev 1999;80:185-189.

19. Nakashima M, Iohara K, Ishikawa M, et al. Stimulation of reparative dentin formation by ex vivo gene therapy using dental pulp stem cells electrotransfected with growth/differentiation factor 11 (Gdf11). Hum Gene Ther 2004;15:1045-1053.

20. Nakashima M, Mizunuma K, Murakami T, Akamine A. Induction of dental pulp stem cell differentiation into odontoblasts by electroporation-mediated gene delivery of growth/differentiation factor 11 (Gdf11). Gene Ther 2002;9:814-818.

21. Lee YS, Lee SJ. Roles of GASP-1 and GDF-11 in dental and craniofacial development. J Oral Med Pain 2015;40:110-114.

22. McPherron AC, Huynh TV, Lee SJ. Redundancy of myostatin and growth/differentiation factor 11 function. BMC Dev Biol 2009;9:24.

23. Walker RG, Poggioli T, Katsimpardi L, et al. Biochemistry and biology of GDF11 and myostatin: similarities, differences, and questions for future investigation. Circ Res 2016;118:1125-1141; discussion 1142.

24. Yung LM, Yang P, Joshi S, et al. ACTRIIA-Fc rebalances activin/ GDF versus BMP signaling in pulmonary hypertension. Sci Transl Med 2020;12:eaaz5660.

25. Yu X, Chen X, Zheng XD, et al. Growth differentiation factor 11 promotes abnormal proliferation and angiogenesis of pulmonary artery endothelial cells. Hypertension 2018;71:729-741.

26. Zhang YH, Cheng F, Du XT, et al. GDF11/BMP11 activates both $\operatorname{smad1/5/8}$ and $\operatorname{smad} 2 / 3$ signals but shows no significant effect on proliferation and migration of human umbilical vein endothelial cells. Oncotarget 2016;7:12063-12074.

27. Jani P, Liu C, Zhang H, Younes K, Benson MD, Qin C. The role of bone morphogenetic proteins 2 and 4 in mouse dentinogenesis. Arch Oral Biol 2018;90:33-39.

28. Nakashima M. Induction of dentine in amputated pulp of dogs by recombinant human bone morphogenetic proteins-2 and -4 with collagen matrix. Arch Oral Biol 1994;39:1085-1089. 